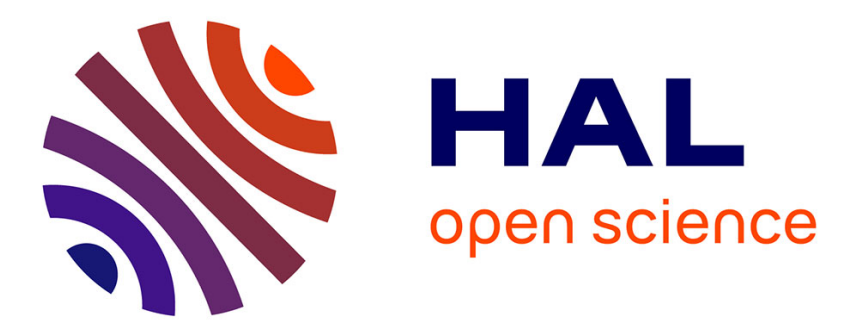

\title{
Microporous Polymers as Macroligands for Pentamethylcyclopentadienylrhodium Transfer-Hydrogenation Catalysts
}

F. Wisser, Y. Mohr, E. Quadrelli, D. Farrusseng, J. Canivet

\section{- To cite this version:}

F. Wisser, Y. Mohr, E. Quadrelli, D. Farrusseng, J. Canivet. Microporous Polymers as Macroligands for Pentamethylcyclopentadienylrhodium Transfer-Hydrogenation Catalysts. ChemCatChem, 2018, 10 (8), pp.1778-1782. 10.1002/cctc.201701836 . hal-01790585

\section{HAL Id: hal-01790585 \\ https://hal.science/hal-01790585}

Submitted on 30 Jul 2020

HAL is a multi-disciplinary open access archive for the deposit and dissemination of scientific research documents, whether they are published or not. The documents may come from teaching and research institutions in France or abroad, or from public or private research centers.
L'archive ouverte pluridisciplinaire HAL, est destinée au dépôt et à la diffusion de documents scientifiques de niveau recherche, publiés ou non, émanant des établissements d'enseignement et de recherche français ou étrangers, des laboratoires publics ou privés. 


\title{
Microporous polymers as macroligands for $\mathrm{Cp}$ * $\mathrm{Rh}$ transfer hydrogenation catalysts
}

\author{
Florian M. Wisser, ${ }^{*[a]}$ Yorck Mohr, ${ }^{[a]}$ Elsje Alessandra Quadrelli, ${ }^{[b]}$ David Farrusseng, ${ }^{[a]}$ Jérôme \\ Canivet*[a]
}

\begin{abstract}
Here we report the synthesis of two bipyridine-based porous polymers and their use as platform for the heterogenization of $\mathrm{Cp}{ }^{\star} \mathrm{Rh}$ catalytic species. These highly stable heterogeneous catalysts demonstrate their efficiency in the transfer hydrogenation of $\alpha$-aryl ketones. Moreover, we show that their catalytic activity is similar to that of homogeneous analogues, highlighting the absence of diffusion limitation in the solids. We rationalize the differences in the measured activities using the Hammett parameter as descriptor of the electronic environment of the catalytic site in both homogeneous and heterogeneous systems.
\end{abstract}

Heterogeneous catalysis allows to circumvent the problem of separation from the products and to simplify the recyclability of the catalyst. One strategy for the heterogeneous catalyst design consists in the integration or grafting of a well-defined molecular catalyst onto a solid support (heterogenization). ${ }^{[1]}$ Grafting active metal catalysts onto a surface allows also for site isolation, thus minimizing interaction and preventing possible multi-metallic deactivation. ${ }^{[2]}$ However, restrictions appear when the integration of the catalytically active centers into a solid support leads to lower performances compared to the homogeneous analogues. ${ }^{[1 a}$, 1e, 2-3]

Molecularly defined support designed as a macroligand, i.e. a large organic or hybrid structure acting like the ligand in molecular
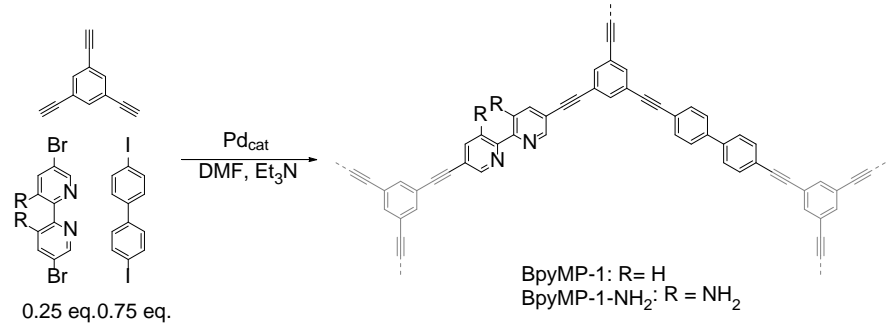

Scheme 1. Synthesis of Cp*Rh@BpyMP microporous catalysts.

[a] Dr. F. M. Wisser, Y. Mohr, Dr. D. Farrusseng, Dr. J. Canivet Univ. Lyon, Université Claude Bernard Lyon 1, CNRS, IRCELYON UMR 5256

2 Av. Albert Einstein, 69626 Villeurbanne, France

E-mail: jerome.canivet@ircelyon.univ-lyon1.fr; florian.wisser@ircelyon.univ-lyon1.fr

[b] Dr. E. A. Quadrelli

Univ. Lyon, Université Claude Bernard Lyon 1, CPE Lyon, CNRS, C2P2 - UMR 5265

43 Bvd. du 11 Novembre 1918, 69616 Villeurbanne, France

Supporting information for this article is given via a link at the end of the document. complex, appears as an appealing opportunity to benefit from advantages of both homogeneous and heterogeneous catalysis. In this perspective, porous frameworks made by the repetition of a coordinating motif, like bipyridine (Bpy) based Metal-Organic Frameworks (MOF) hybrids and purely organic conjugated microporous polymers (CMP), are promising candidate platforms for molecular catalysts heterogenization.

Conjugated microporous polymers are 3D amorphous materials, first introduced by Cooper and co-workers. ${ }^{[4]}$ Their synthesis by classical carbon-carbon coupling reactions allow for the integration of metal binding sites such as bipyridines. They have been applied as host material for the fixation of $\mathrm{Re}$ and $\mathrm{Ru}$ for photocatalysis, ${ }^{[5]} \mathrm{Pd}$ for $\mathrm{C}-\mathrm{C}$ coupling reactions ${ }^{[6]}$ and recently Ir for formate decomposition. ${ }^{[7]}$

Here we present new CMP derivatives made by using Bpybased monomers to synthesise copolymers, named hereafter Bipyridine Microporous Polymers (BpyMP) materials. They were used as platforms for the site isolation of organometallic $\mathrm{Cp}^{\star} \mathrm{Rh}$ complexes (Scheme 1). These catalysts were found to be active in the transfer hydrogenation of $\alpha$-aryl ketones. Comparing the catalytic activity of our solids to that obtained using their molecular analogues, we found that no diffusion limitation occurs under the reported conditions.

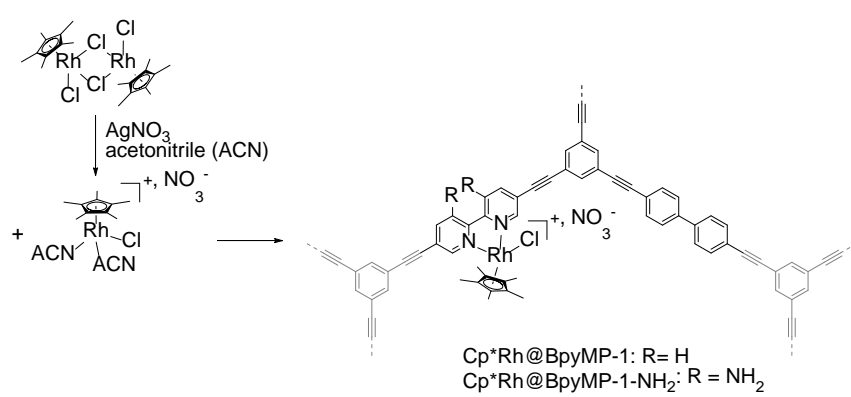

The BpyMP-1 platform was prepared by copolymerization of 1,3,5-triethynylbenzene with 5,5'-dibromo-2,2'-bipyridine (25 mol-\%) and 4,4'-diiodo-biphenyl (75 mol-\%) (Scheme 1) in the presence of palladium catalyst (2 mol\%). ${ }^{[8]}$ BpyMP-1- $\mathrm{NH}_{2}$ was obtained similarly by replacing $5,5^{\prime}$-dibromo-2,2'-bipyridine by 3,3'-diamino-5,5'-dibromo-2,2'-bipyridine. The functionalization with the rhodium precursor was performed by infiltration with a solution of $\left[\mathrm{Cp}^{\star} \mathrm{RhCl}\right] \mathrm{NO}_{3}$ (16 mol\% with respect to Bpy moieties) to reach an identical $\mathrm{Rh}$ loading of $1.6 \mathrm{wt} \%$, as determined by ICPOES analysis, in the respective Cp*Rh@BpyMP-1 and $\mathrm{Cp}{ }^{\star} \mathrm{Rh} @ B p y M P-1-\mathrm{NH}_{2}$ solids. Elemental analysis also reveals that less than $0.5 \mathrm{wt} \%$ of $\mathrm{Pd}$ remains in the final materials. 
Complete copolymerization of bipyridine- and biphenyl-based monomers with triethynylbenzene was confirmed by IR spectroscopy (Figure 1, Figure S1). No band at $2100 \mathrm{~cm}^{-1}$ from the terminal acetylene groups is observed. Instead a strong band at $2200 \mathrm{~cm}^{-1}$ can be detected in all porous polymers corresponding to the asymmetric stretching vibration of medial triple bonds. ${ }^{[9]}$ The presence of 1,3,5-trisubstituted and parasubstituted benzene moieties is confirmed by the broad vibrations around 1580 and $1490 \mathrm{~cm}^{-1}$ as well as by the strong band at $820 \mathrm{~cm}^{-1}$ for the para-substituted benzene (polymerized biphenyl). ${ }^{[10]}$ Bands at 1450 and $1435 \mathrm{~cm}^{-1}$ are attributed to $\mathrm{C}-\mathrm{H}$ deformation vibrations of the bipyridine moieties and the band at $1260 \mathrm{~cm}^{-1}$ to $\mathrm{C}-\mathrm{N}$ stretching vibration of aromatic amines in BpyMP-1- $\mathrm{NH}_{2} \cdot{ }^{[9,11]}$

In ${ }^{13} \mathrm{C}$ MAS NMR spectra, signals around 140, 130 and $125 \mathrm{ppm}$ can be attributed to the aromatic carbon atoms of the backbone (Figure S2 and S3). The signal at 90 ppm belongs to medial triple bonds, whereas no signal of carbon atoms from terminal triple bonds can be detected around $80 \mathrm{ppm}$. This further confirms the high degree of polymerization. The signals at 95 and $10 \mathrm{ppm}$ in the spectra of the $\mathrm{Cp}^{*} \mathrm{Rh}$ loaded samples belongs to the quaternary and $\mathrm{CH}_{3}$ carbon atoms of the pentamethylcyclopentadienyl moiety respectively.

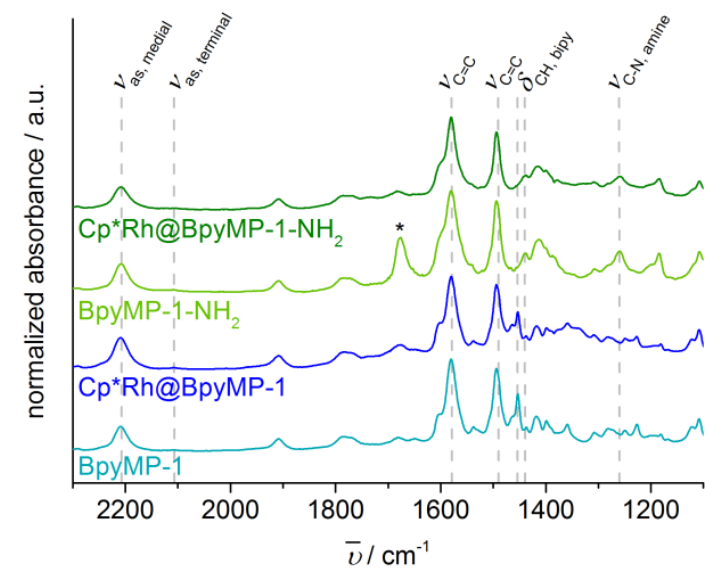

Figure 1. IR spectra of BpyMP-1 (cyan), 1.6\%Cp*Rh@BpyMP-1 (blue), BpyMP1- $\mathrm{NH}_{2}$ (pale green) and 1.6\%Cp*Rh@BpyMP-1- $\mathrm{NH}_{2}$ (green). * denotes band form remaining DMF.

Nitrogen physisorption isotherms recorded for both the pristine polymers and the Rh-functionalized ones show a hysteresis, characteristic for the swelling behaviour of porous polymers (Figure S5). The apparent surface area ${ }^{[12]}$ determined by the BET method for BpyMP-1 and BpyMP-1-NH $\mathrm{NH}_{2}$ was $680 \pm 30$ $\mathrm{m}^{2} / \mathrm{g}$ and $700 \pm 20 \mathrm{~m}^{2} / \mathrm{g}$, respectively. As expected the porosity slightly decreases upon $\mathrm{Cp} * \mathrm{Rh}$ insertion to $570 \pm 30 \mathrm{~m}^{2} / \mathrm{g}$ for Cp*Rh@BpyMP-1 and $680 \pm 10$ m²/g for Cp*Rh@BpyMP-1- $\mathrm{NH}_{2}$.

Having these novel $\mathrm{Cp}{ }^{*} \mathrm{Rh}(\mathrm{bpy})$-based porous polymers in hand, we investigated their catalytic potential in the $\alpha$-aryl ketones transfer hydrogenation to give the corresponding alcohols using isopropanol as hydrogen donor (Scheme 2 ). We note that under conditions reported in literature, e.g. at $82^{\circ} \mathrm{C}$ in ${ }^{i} \mathrm{PrOH} /{ }^{i} \mathrm{PrOK},{ }^{[13]}$ the transfer hydrogenation of acetophenone occurs even without any catalyst (40\% conversion after 18 hours). We thus investigated this reaction under smoother conditions and we accordingly reduced the temperature to $60^{\circ} \mathrm{C}$, consequently lowering the catalytic activity.
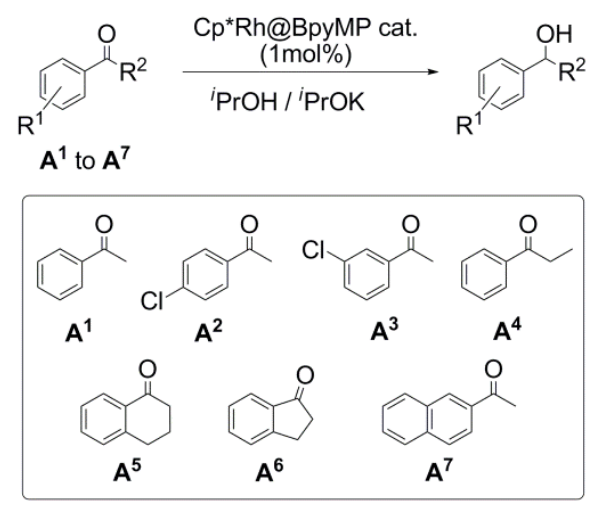

Scheme 2. Transfer hydrogenation a-aryl ketones $A^{1}$ to $A^{7}$ catalyzed by Cp*Rh@BpyMP catalysts.

The BpyMP-based heterogeneous catalysts were compared to homogeneous counterparts based on 2,2'-bipyridine (bpy) and 5,5'-bis(phenylethynylene)-2,2'-bipyridine (bpebpy) ligand, the latter being the closest molecular analogue to BpyMP-1.

We note that the palladium, remaining from the synthesis, in the BpyMP structures is not active, as the same conversion $(<1 \%$ after 3 h) can be observed over the pristine BpyMP-1 network and in a reaction performed without any catalyst (Table 1, entries 1 and 2).

Table 1. Transfer hydrogenation of $\alpha$-aryl ketones into alcohols catalyzed by Cp*Rh@BpyMP-1, Cp*Rh@BpyMP-1-NH $\mathrm{N}_{2}$ and molecular analogues. ${ }^{\text {[a] }}$

\begin{tabular}{|c|c|c|c|}
\hline Entry & Catalyst & Ketone & TOF $\left(h^{-1}\right)^{[b]}$ \\
\hline 1 & none & $A^{1}$ & $(X=0.8 \%)^{[c]}$ \\
\hline 2 & BpyMP-1 (without Rh) & $A^{1}$ & $(X=0.5 \%)^{[c]}$ \\
\hline 3 & $\mathrm{Cp}^{\star} \mathrm{Rh}(\mathrm{bpy}) \mathrm{Cl}_{2}$ & $A^{1}$ & $2.66 \pm 0.13$ \\
\hline 4 & $\mathrm{Cp}^{*} \mathrm{Rh}\left(\right.$ bpebpy)Cl${ }_{2}$ & $A^{1}$ & $1.55 \pm 0.08$ \\
\hline 5 & Cp*Rh@BpyMP-1 & $A^{1}$ & $1.76 \pm 0.09$ \\
\hline 6 & Cp*Rh@BpyMP-1-NH ${ }_{2}$ & $A^{1}$ & $2.96 \pm 0.15$ \\
\hline 7 & Cp*Rh@BpyMP-1-NH & $A^{2}$ & $2.48 \pm 0.12$ \\
\hline 8 & Cp*Rh@BpyMP-1-NH & $A^{3}$ & $3.32 \pm 0.16$ \\
\hline 9 & Cp*Rh@BpyMP-1-NH & $A^{4}$ & $0.90 \pm 0.05$ \\
\hline 10 & Cp*Rh@BpyMP-1-NH & $A^{5}$ & $0.67 \pm 0.03$ \\
\hline 11 & Cp*Rh@BpyMP-1-NH 2 & $A^{6}$ & $0.44 \pm 0.10$ \\
\hline 12 & Cp*Rh@BpyMP-1-NH & $A^{7}$ & $2.03 \pm 0.19$ \\
\hline
\end{tabular}

[a] Reaction conditions: $60^{\circ} \mathrm{C}$, molar ratio of Ketone / KOH / catalyst: $100 / 10$ / 1 in $\mathrm{PrOH}$. [b] Initial TOF determined after 3 hours and expressed as mol of product/(mol of $\mathrm{Rh}$. time). [c] Conversion $X$ after 3 hours are given in parentheses in the absence of $\mathrm{Cp}{ }^{\star} \mathrm{Rh}$. 
Both homogeneous and heterogeneous catalytic systems were found to efficiently catalyze the reduction of acetophenone $\left(A^{1}\right)$ into 1-phenyl-ethanol (Table 1 , entries $3-6$ ). The highest TOF $\left(\sim 3 \mathrm{~h}^{-1}\right)$ for the conversion of acetophenone into 1-phenylethanol was achieved using $\mathrm{Cp}{ }^{\star} \mathrm{Rh} @ B$ ByMP-1-NH

For all homogeneous and heterogeneous catalysts a slow deactivation occurs over time resulting in a decrease in activity after three hours (see Figure S9). We note that the most active heterogenized catalyst Cp* $\mathrm{Rh} @ B p y M P-1-\mathrm{NH}_{2}$ could be reused for 3 times without a significant decrease in activity after $3 \mathrm{~h}$ of reaction.

For the most active catalyst, namely Cp*Rh@BpyMP-1-NH $\mathrm{N}_{2}$, we evaluated different $\alpha$-aryl ketones as substrates. As shown in the Table 1, similar activities can be observed for substituted acetophenones, with the highest activity reaching $3.3 \mathrm{~h}^{-1}$ in the case of 2-chloro-acetophenone ( $\mathbf{A}^{3}$, entry 6$)$. The catalytic activity is significantly decreased below $1 \mathrm{~h}^{-1}$ when cyclic ketones or longer alkyl chain ketones were used as substrates $\left(\mathbf{A}^{4}\right.$ to $\mathbf{A}^{6}$ in entries 7 - 9). However we can exclude major diffusion limitation within the porous macroligand as for the bulkier 2acetonaphthone ( $\mathbf{A}^{7}$, entry 10$)$ a TOF of $2.0 \mathrm{~h}^{-1}$ was achieved. $A$ relative rigidity of the substrate at the reactive $\mathrm{C}=\mathrm{O}$ bond, like in cyclic ketones $A^{5}$ and $A^{6}$, might hinder the formation of $R h$ transition species, lowering the corresponding activity. Similarly, having a quite symmetrical bulkiness around the $\mathrm{C}=\mathrm{O}$ bond, like in the ethyl phenyl ketone $\mathbf{A}^{4}$, could limit the approach of the substrate to the Rh active center. This hindering effect might further be enhanced by the steric constraints at the molecular level imposed by the cavity walls.

The $C p^{*} \mathrm{Rh} @$ BpyMP-1-NH $\mathrm{H}_{2}$ is found to be stable after recycling for 3 runs using $A^{1}$ as substrate, a slight decrease in activity is observed during the fourth run (Figure S10). This reduced activity might arise from pore blocking due to base accumulation during recycling process (Figures S14 to S17 and associated discussion).

To evaluate the influence of the different bpy-substituents on the activity of heterogeneous systems, we extended the scope of host frameworks also to bpy-based MOFs, namely UiO-67-bpy and MOF-253 (see Supporting Information for detailed characterizations). Both MOFs has been already applied in different catalytic reaction as macroligands, including $\mathrm{Cp}^{\star} \mathrm{Rh}$ functionalized ones. ${ }^{[14]}$ The Cp*Rh@UiO-67-bpy and Cp*Rh@MOF-253, containing similar Rh loadings (ca. 2 wt\%) both show lower TOF values $\left(0.698 \pm 0.035 \mathrm{~h}^{-1}\right.$ and $0.455 \pm 0.023$ $\mathrm{h}^{-1}$ respectively) than BpyMP-based catalysts for the transfer hydrogenation of the acetophenone.

The comparison of the different heterogeneous macroligands was realized by applying the Hammett-correlation. Such a correlation is well established for homogeneous catalysts but has not yet been reported for heterogeneous organometallic- or organocatalysts.

Therefore, in the case of acetophenone susbtrate, we plotted the logarithm of the ratio between the TOF of a selected (macro)ligand with a substituent $\mathrm{R}$ with respect to the TOF obtained with bipyridine used as reference ligand $\left(R=H, \sigma_{H}=0\right)$ against its Hammett constant $(\sigma)$ :

$$
\lg \left(\frac{T O F_{\mathrm{R}}}{T O F_{\mathrm{R}=\mathrm{H}}}\right)=\rho \cdot \sigma_{\mathrm{R}}
$$

with $\sigma_{R}$ being the Hammett constant of the substituent R and $\rho$ the reaction constant which depends on the reaction conditions, e.g. solvent or temperature. Hammett constant values were attributed to the corresponding $\mathrm{R}$ substituents according to literature. ${ }^{[15]}$

Here we found a unique linear correlation followed by both homogeneous and heterogeneous catalysts, covering Hammett constants from -0.02 (for meta-amino in BpyMP1- $\mathrm{NH}_{2}$ ) to 0.66 (for meta-aluminum carboxylate in MOF-253) (Figure 2). The fact that heterogeneous BpyMP and MOF-based catalysts follow the same linear correlation than homogeneous molecular species proves that the performance of the catalysts depends only on the substituents electronic properties for both homogeneous and heterogeneous systems, further excluding any diffusion limitation inside these porous materials. The low Hammett constant of the BpyMP-1- $\mathrm{NH}_{2}$ based catalyst give rise to the highest electron density on the rhodium, ${ }^{[16]}$ which causes the highest activity of this catalyst in the transfer hydrogenation reaction.

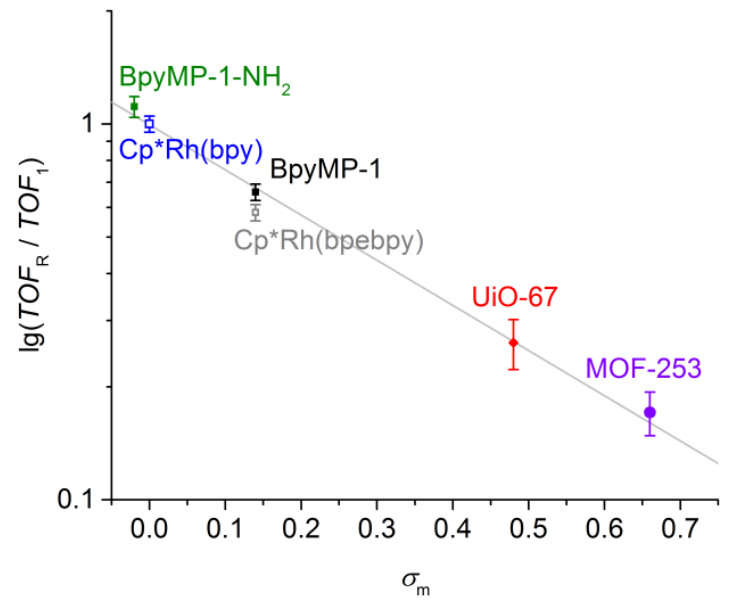

Figure 2. Hammett plot of TOF of transfer hydrogenation reaction of acetophenone into 1-phenyl-ethanol over different homogeneous and heterogeneous catalysts.

In conclusion we introduced bipyridine containing Microporous Polymers as novel macroligand platforms for efficient heterogenization of organometallic catalysts. The activity of heterogeneous $\mathrm{Cp}{ }^{\star} \mathrm{Rh}$ catalytic centers can be tuned by rationally choosing adequate functional pending groups onto such macroligands, as demonstrated here for the transfer hydrogenation of $\alpha$-aryl ketones. The absence of diffusion limitation inside the porous system and a catalytic behavior identical to that of molecular analogues make BpyMP very appealing heterogeneous macroligand for organometallic catalysts. The good correlation between homogeneous and heterogeneous catalyst highlights also the preservation of the electronic environment around the active site upon heterogenization. The obtained catalysts are long range solids but can be considered as molecularly analogous to homogeneous complexes when it comes to catalysis. Such species further bring closer homogeneous and heterogeneous catalysis. 


\section{Experimental Section}

Synthesis of BpyMP catalysts. The synthesis of BpyMP materials was done according to a modified literature procedure. ${ }^{[8]}$

For a typical synthesis of BpyMP-1, $100.0 \mathrm{mg}$ (0.66 mmol) 1.3.5triethynylbenzene, $310.1 \mathrm{mg}$ ( $0.75 \mathrm{mmol})$ 4,4'-diiodobiphenyl, $86.0 \mathrm{mg}$ (0.25 mmol) 5,5'-dibromo-2,2'-bipyridine and $15.7 \mathrm{mg} \quad(13.4 \mu \mathrm{mol})$ $\mathrm{Pd}\left(\mathrm{PPh}_{3}\right)_{4}$ were added to a flame dried glass flask inside a glove box. The glass vial was sealed with a teflon septum. Outside the glove box, $12 \mathrm{ml}$ of anhydrous DMF and $6 \mathrm{ml}$ of anhydrous $\mathrm{NEt}_{3}$ were added and the glass vial was placed in an oil bath. The reaction mixture was heated to $100{ }^{\circ} \mathrm{C}$ for $24 \mathrm{~h}$. After cooling down to room temperature the yellow solid was purified by soxhlet extraction using $\mathrm{CHCl}_{3}$ for $6 \mathrm{~h}$ and using $\mathrm{MeOH}$ overnight.

For a typical infiltration, (6 mol-\% Rh) 100 mg BpyMP-1 were dispersed in $3.7 \mathrm{ml}$ dry $\mathrm{MeOH}$, then $1.05 \mathrm{ml}$ of $15 \mu \mathrm{mol} / \mathrm{ml}\left[\mathrm{Cp}^{*} \mathrm{RhCl}^{\mathrm{N}} \mathrm{NO}_{3}\right.$ in acetonitrile were added, and the suspension was stirred for $24 \mathrm{~h}$ at room temperature to yield $1.6 \% \mathrm{Cp} \mathrm{Rh}^{\star} @ \mathrm{BpyMP}-1$. The supernatant was removed by centrifugation and the yellow solid washed with $\mathrm{MeOH}$ for 2 days.

Catalysis. Transfer hydrogenation was carried out under Ar atmosphere in glass vials containing approximately $2.2 \mu \mathrm{mol}$ catalyst in $2 \mathrm{ml}$ of $0.011 \mathrm{mM}$ deaerated $\mathrm{KOH}$ solution in 2-PrOH. After activating the catalyst for $1 \mathrm{~h}$ at $60^{\circ} \mathrm{C} 0.21 \mathrm{mmol}$ ketone were added and the reaction mixture heated to $60^{\circ} \mathrm{C}$ for $24 \mathrm{~h}$. After certain time intervals $50 \mu \mathrm{l}$ of the reaction

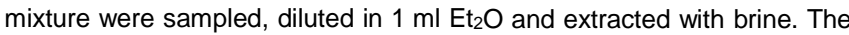
organic phase was analyzed by HPLC using a Shimadzu LC 2010 liquid chromatograph equipped with a AS-H column (isocratic mode: $n$-hexane : 2-PrOH, $97: 3, V: V$, detection @ $215 \mathrm{~nm}$ ). To estimate an error the largest relative error determined from two consecutive measurements of the same sample was applied to all data given.

\section{Acknowledgements}

F.M.W. gratefully acknowledges financial support from the Deutsche Forschungsgemeinschaft (DFG, Postdoctoral Research Fellowship, grant number WI 4721/1-1). The authors are very grateful to Dr. L. Cardenas for XPS analysis, C. Lorentz for slide state NMR spectroscopy and N. Cristin and P. Mascunan for ICP-OES analysis.

Keywords: Microporous Polymers • Heterogenization • Transfer Hydrogenation $\bullet$ Hammett parameter

[1] a) L.-N. He, J.-Q. Wang, J.-L. Wang, Pure Appl. Chem. 2009, 81, 20692080; b) M. Rose, R. Palkovits, in Metal Organic Frameworks as Heterogeneous Catalysts (Eds.: F. X. Llabrés i Xamena, J. Gascon), RSC Catalysis Series no 12, RSC Publishing, Cambridge, 2013, pp. 384-
405; c) M. P. Conley, C. Copéret, Top. Catal. 2014, 57, 843-851; d) A. G. Slater, A. I. Cooper, Science 2015, 348; e) G. H. Gunasekar, K. Park, K. D. Jung, S. Yoon, Inorg. Chem. Front. 2016, 3, 882-895.

[2] X. Liu, S. Inagaki, J. Gong, Angew. Chem. Int. Ed. 2016, 55, 14924 14950.

[3] a) E. A. Quadrelli, J.-M. Basset, Coord. Chem. Rev. 2010, 254, 707-728: b) L. Wang, F.-S. Xiao, ChemCatChem 2014, 6, 3048-3052.

[4] J.-X. Jiang, F. Su, A. Trewin, C. D. Wood, N. L. Campbell, H. Niu, C. Dickinson, A. Y. Ganin, M. J. Rosseinsky, Y. Z. Khimyak, A. I. Cooper Angew. Chem., Int. Ed. 2007, 46, 8574-8578.

[5] a) R. R. Haikal, X. Wang, Y. S. Hassan, M. R. Parida, B. Murali, O. F. Mohammed, P. J. Pellechia, M. Fontecave, M. H. Alkordi, ACS Appl. Mater. Interfaces 2016, 8, 19994-20002; b) J.-X. Jiang, C. Wang, A. Laybourn, T. Hasell, R. Clowes, Y. Z. Khimyak, J. Xiao, S. J. Higgins, D. J. Adams, A. I. Cooper, Angew. Chem. Int. Ed. 2011, 50, 1072-1075; c) W. Liang, T. L. Church, S. Zheng, C. Zhou, B. S. Haynes, D. M. D'Alessandro, Chem. - Eur. J. 2015, 21, 18576-18579; d) C. Wang, Z. Xie, K. E. deKrafft, W. Lin, ACS Appl. Mater. Interfaces 2012, 4, 22882294

[6] C.-A. Wang, Y.-F. Han, Y.-W. Li, K. Nie, X.-L. Cheng, J.-P. Zhang, RSC Adv. 2016, 6, 34866-34871.

[7] C. Broicher, S. Foit, M. Rose, P. J. C. Hausoul, R. Palkovits, ACS Catal. 2017, 7, 8413-8419.

[8] M. Trunk, A. Herrmann, H. Bildirir, A. Yassin, J. Schmidt, A. Thomas Chem. - Eur. J. 2016, 22, 7179-7183.

[9] J. Coates, in Encyclopedia of Analytical Chemistry (Ed.: R. A. Meyers) John Wiley \& Sons Ltd, Chichester, 2000, pp. 10815-10837.

[10] a) S. Sandroni, F. Geiss, Fresenius' Z. Anal. Chem. 1966, 220, 321-333; b) M. V. Shishkina, M. M. Teplyakov, V. P. Chebotaryev, V. V. Korshak Makromol. Chem. 1974, 175, 3475-3485.

[11] E. Castellucci, L. Angeloni, N. Neto, G. Sbrana, Chem. Phys. 1979, 43 365-373.

[12] R. Dawson, A. I. Cooper, D. J. Adams, Prog. Polym. Sci. 2012, 37, 530 563.

[13] J. M. Gichumbi, H. B. Friedrich, B. Omondi, J. Mol. Catal. A: Chem. 2016 416, 29-38.

[14] a) C. Wang, Z. Xie, K. E. deKrafft, W. Lin, J. Am. Chem. Soc. 2011, 133 13445-13454; b) D. Sun, Y. Gao, J. Fu, X. Zeng, Z. Chen, Z. Li, Chem. Commun. 2015, 51, 2645-2648; c) M. B. Chambers, X. Wang, N. Elgrishi, C. H. Hendon, A. Walsh, J. Bonnefoy, J. Canivet, E. A. Quadrelli, D. Farrusseng, C. Mellot-Draznieks, M. Fontecave, ChemSusChem 2015, 8, 603-608; d) T. Kajiwara, M. Fujii, M. Tsujimoto, K. Kobayashi, M. Higuchi, K. Tanaka, S. Kitagawa, Angew. Chem., Int. Ed. 2016, 55, 2697 2700; e) M. Rimoldi, A. J. Howarth, M. R. DeStefano, L. Lin, S. Goswami, P. Li, J. T. Hupp, O. K. Farha, ACS Catalysis 2017, 7, 997-1014; f) M. I. Gonzalez, J. Oktawiec, J. R. Long, Faraday Discuss. 2017, 201, 351-367.

[15] C. Hansch, A. Leo, R. W. Taft, Chem. Rev. 1991, 91, 165-195.

[16] a) Y. Himeda, S. Miyazawa, T. Hirose, Chem SusChem 2011, 4, 487-493; b) Y. Himeda, N. Onozawa-Komatsuzaki, H. Sugihara, K. Kasuga Organometallics 2007, 26, 702-712. 
